\title{
Ecoeficiencia y Gestión Ambiental Sostenible: Reflexiones para la Gerencia del Siglo XXI
}

\section{Eco-efficiency and Sustainable Environmental Management: Reflections for the Management of the 21StCentury}

\author{
Diana Patricia Eljach-Hernandez \\ diana.eliach@urbe.edu.ve \\ Universidad Privada Dr. Rafael Belloso Chacín, Zulia \\ Venezuela \\ https://orcid.org/0000-0002-7184-4134 \\ William Wannergg Castro-Castellanos \\ wcastro@urbe.edu.ve \\ Universidad Privada Dr. Rafael Belloso Chacín, Zulia \\ Venezuela \\ https://orcid.org/0000-0003-0297-9243
}

Recibido: 01 de junio de 2020

Aprobado: 20 de agosto de 2020 


\section{RESUMEN}

El presente estudio tuvo como objetivo analizar la ecoeficiencia y la gestión ambiental sostenible en la gerencia del siglo XXI en el contexto colombiano, sustentándose teóricamente en los autores: Leal (2005), Manjarres y Chirino (2020); Bleischwitz (2003), Aldana Zavala, J. J. (2019), entre otros. Asimismo, se enmarcó como investigación documental, orientada a evaluar la importancia de la ecoeficiencia y la gestión ambiental sostenible como aspectos relevantes para la toma de decisiones de la nueva gerencia. El resultado de esta revisión, infiere que existe la necesidad imperante de enrutar el éxito empresarial mediante la implementación de políticas organizacionales que garanticen la conservación, protección, uso eficiente de los recursos naturales a fin de contribuir mediante acciones que contrapesen el deterioro ambiental del planeta tierra. Si bien es cierto, que esto representa un reto para las organizaciones, también es importante la concientización del legado que se pretende instituir para las generaciones venideras.

Descriptores: Ecoeficiencia; gestión ambiental; gerencia; desarrollo sostenible (Palabras del Tesauro UNESCO).

\section{ABSTRACT}

The present study aimed to analyze eco-efficiency and sustainable environmental management in the management of the XXI century in the Colombian context, theoretically based on the authors: Leal (2005), Manjarres and Chirino (2020); Bleischwitz (2003), Aldana Zavala, J. J. (2019), among others. Likewise, it was framed as a documentary research, aimed at evaluating the importance of eco-efficiency and sustainable environmental management as relevant aspects for the decision-making of the new management. The result of this review infers that there is a prevailing need to guide business success through the implementation of organizational policies that guarantee the conservation, protection, efficient use of natural resources in order to contribute through actions that counterbalance the environmental deterioration of planet earth. While it is true that this represents a challenge for organizations, it is also important to raise awareness of the legacy that is intended to be instituted for future generations.

Descriptors: Eco-efficiency; environmental management; management, sustainable development. (Words from UNESCO Thesaurus). 


\section{INTRODUCCIÓN}

Hace más de dos décadas que en una cumbre mundial sobre Desarrollo Sostenible se escuchó por primera vez el término de ecoeficiencia (Cumbre de Rio de Janeiro 1992, Informe de la Conferencia de las Naciones Unidas sobre el Medio Humano, Estocolmo, 5 a 16 de junio de 1972. Declaración de Río sobre el Medio Ambiente y el Desarrollo La Conferencia de las Naciones Unidas sobre el Medio Ambiente y el Desarrollo), Luego, en la evolución del concepto se le añaden ciertas características tales como: enfatizar en la creación de valor, en la durabilidad, en el largo plazo, en objetivos para el mejoramiento continuo, la vinculación de la excelencia ambiental con la excelencia empresarial y considerar tanto el consumo como la producción sostenible (DeSimone \& Popoff, 1997). De igual forma, en el giro del concepto se incluye un matiz social cuando Mickwitz (2006) lo define como: la eficiencia con la cual los recursos ecológicos son usados para satisfacer las necesidades humanas. Sin embargo, no se trata solo de un concepto abstracto, sino de una política empresarial cuyo comportamiento es medible en los factores económicos, medioambientales, socioculturales y técnicos, tanto en indicadores a corto y largo plazo, como también se puede medir en virtud de la relación que existe entre el valor del producto o servicio y el impacto ambiental; tal como lo expresan Curcelle (1998) \& Erkko, Melanen \& Mickwitz (2005).

Hacer un acercamiento a la afirmación de Kramer \& Porter (2002), quienes hablan del "valor compartido solo puede ser el resultado de una efectiva colaboración entre las partes" (p. 48), transporta a las acciones realizadas desde el aparato productivo que influyen, afectan e incluso generan situaciones sociales, ambientales y económicas, como escenario de malestar en general, cayendo la confianza del sector productivo, aunado al afán mercantilista en la obtención de dividendos financieros, con una visión cortoplacista. Sin embargo, el valor compartido, es una oportunidad de crear valor social, empresarial y ambiental desde el reaprendizaje en pro de lo sistémico que impacte el todo, a través de reinvención de productos y mercados, redefinición de la productividad en la cadena de valor y el desarrollo de clúster 
En otras palabras, es promover un desarrollo cultural que fortalezca las interacciones, las redes sociales y la capacidad de generar sinergias (Ávila, 2012). Cosa diferente a lo visto con el transcurrir del tiempo, cuando permanecemos inmersos en una economía de mercado, tal y como asevera Kliksberg (2000), en dónde con las esperadas altas tasas de crecimiento económico, se obtendrían beneficios que serían socializados a los más vulnerables, disminuyendo el índice de pobreza y se alcanzaría un crecimiento al mismo tiempo, con un desarrollo social y ambiental. Ya se conocen los resultados.

Cuando se ingresa al campo de la gerencia, se entiende esta como la formulación, ejecución y evaluación de acciones que permitirán que una organización alcance sus objetivos trazados y para tal fin debe conocer sus fortalezas, debilidades, amenazas y oportunidades apelando a las diferentes estrategias para este fin. Es ahí donde se debe acoger un modelo de desarrollo sostenible que apunte a ser "aquel que satisface las necesidades de la presente generación sin comprometer las necesidades de las futuras generaciones" (Comisión Mundial del Medio Ambiente y del Desarrollo,1988).

Teniendo en cuenta lo anterior, se procede a realizar una serie de reflexiones acerca de la ecoeficiencia y de la gestión ambiental sostenible para la gerencia del siglo XXI, las cuales permitirán dar respuestas a las preguntas que se enuncian a continuación ¿Por qué la ecoeficiencia y la gestión ambiental sostenible representan un reto para la gerencia del siglo XXI? ¿Qué motiva a las empresas a incorporar estas políticas a sus prácticas? ¿Qué nos depara en el futuro si se decide ignorar tan importante temática? Es preciso denotar que, en los países industrializados la situación en términos medioambientales es diferente a la de los países en vías de desarrollo, razón por la cual los proveedores, los clientes y la misma organización hacen parte de un proceso que lidera los mercados internacionales, pues las condiciones favorables de políticas públicas solidas han permitido la migración a un estado de mayor conciencia en el uso racional de los recursos naturales en la medida que se satisfacen las necesidades humanas. 
Ahora bien, el reto para la gerencia del siglo XXI debe ir de la mano de los planes de desarrollo de cada país, estableciendo así un paralelo entre la normatividad y la concientización real a partir de la gestión del conocimiento, del manejo de materia primas, uso de tecnología en el procesamiento, transformación y distribución del producto, manejo de desechos, emisión de gases contaminantes, energía eléctrica sostenible entre otros; de tal manera que las políticas no se conviertan en letra muerta si no sean el soporte para una nueva visión organizacional que asegura el bienestar a las próximas generaciones.

Adentrarse al mundo actual, es reconocer que hay cambios trascendentales desde la mirada del proceso globalizador, en donde las relaciones pasan de ser unidireccionales a integrales. Para comprender, entender y actuar ante esta nueva realidad, se hace necesario nuevas maneras de ver la vida, las relaciones, las interrelaciones y más allá de los paradigmas establecidos. Por lo cual una visión holística, ecológica e innovadora debe ser capaz de gerenciar estos cambios y circunstancias cotidianas que se presentan. He ahí el verdadero reto de la Gerencia, asumir lo desconocido, disminuir el nivel de incertidumbre para la toma de decisiones siempre en pro de las nuevas generaciones y su calidad de vida, conservando nuestra naturaleza.

A través de los años, el concepto de demanda en el mercado ha variado, ya el consumidor no se mantiene apacible ante la situación de la sociedad, es una época de cambios disruptivos, de la aplicación del pensamiento sistémico y la aparición de nuevas formas de dirigir, empoderar y generar nuevos canales de comunicación con el entorno y la sociedad. Con este panorama en cierne, las necesidades del mercado a suplir, no pasan única y exclusivamente por lo primario, hoy intervienen otros factores, entre ellos el ambiental, el social y la innovación que logre satisfacer los sentidos y las emociones. Siendo este el panorama, no podremos obtener mejores resultados haciendo lo mismo; es hora de cambiar para mejorar.

En la era en donde las organizaciones no solo aprenden, sino desaprenden y reaprenden comportamientos, maneras de actuar y accionar ante sus clientes internos y 
externos, nos encontramos con organizaciones que se autoevalúan, autogeneran y están en permanente mejoramiento continuo. De tal manera que, lo único constante es el cambio, siendo así, el estar quieto en un mar de nuevas opciones que involucra ecoeficiencia, gestión ambiental sostenible y gerencia, no puede ser otro el camino que ir en busca de la embarcación del cumpliendo de los objetivos de desarrollo sostenible que, desde una mirada empresarial, sopena de adentrarnos a una gran travesía o quedarnos a que la inanición nos alcance. Hecha estas consideraciones, es necesario analizar la ecoeficiencia y la gestión ambiental sostenible en la gerencia del siglo XXI dentro del contexto colombiano.

\section{METODOLOGÍA.}

Esta investigación se encuentra definida por el tipo de estudio y la fuente de información en la que se encuentra enmarcada. En este sentido, el tipo de investigación es documental. Al respecto, Palella y Martins (2012), la definen como aquella que se concreta en la recopilación de información en diversas fuentes documentales escritas u orales, teniéndose como población de estudio y a modo de unidades de análisis, textos, artículos científicos, documentos oficiales, entre otros. Bajo esta premisa se analizaron fuentes digitales, manuales, así como textos con el fin de establecer los criterios que orientaron el presente estudio y que permitieron considerar los elementos desde una perspectiva analítica que dio paso a un proceso reflexivo por parte de los investigadores (Aldana Zavala, 2019). 


\section{ANÁLISIS Y DISCUSIÓN}

Para nadie es un secreto que nuestro planeta tierra está sufriendo un deterioro cada vez más marcado. Las proyecciones indican que deben tomarse medidas de manera inmediata para evitar el colapso mundial. Algunos lineamientos provienen de las Naciones Unidas, así como de la comunidad internacional, donde cada país adopta los programas, protocolos o medidas que contribuyan con la minimización del cambio climático.

Al mismo tiempo, todas estas estrategias impactan de alguna manera a las grandes, medianas y pequeñas empresas, las cuales han tenido que repensar la forma como ejecutan sus procesos en cado uno de los departamentos o áreas que la integran (dirección, administración, ventas, producción, contabilidad y finanzas, entre otras) teniendo en cuenta las directrices internacionales que apuntan siempre a la competitividad global.

Por su parte las instituciones educativas realizan un proceso de formación que es fundamental, puesto que las nuevas generaciones van de la mano con el cuidado y protección del ambiente en el sentido de que sus acciones se encaminan a salvaguardarlo. Es por eso que las cátedras universitarias promueven soluciones que sean amigables y sostenibles a situaciones ambientales en detrimento. Estas condiciones son diferentes a las que se viven actualmente, puesto que muchas de las organizaciones les toco cambiar sobre la marcha, ya que en sus inicios no fueron creadas para optimizar procesos de manera ecoeficiente, si no que se atendía a una necesidad de margen de rentabilidad.

Teniendo en cuenta lo anterior, las organizaciones que pretenden alcanzar mercados internacionales, han invertido en una planeación estratégica innovadora, direccionamiento de vanguardia, tecnología de última generación, cuya implementación garantice un mínimo de impacto ambiental con el máximo de optimización de los recursos. Esto, las ha llevado a incorporar dentro de su filosofía y su estrategia 
corporativa fundamentos y orientadores en relación a la Ecoeficiencia y la Gestión Ambiental Sostenible

\section{Ecoeficiencia y Gestión Ambiental Sostenible}

El termino de ecoeficiencia proviene del concepto de ecología según el Tesauro de la UNESCO, fue presentada en la literatura académica por Schaltegger y Sturm (1990) y Schaltegger y Burritt, (2000). Sin embargo, Schmidheiny (1992) popularizó el término a partir del cual ganó reconocimiento en las agendas globales empresariales, en especial las lideradas por el World Business Council for Sustainable Development (WBCSD) por sus siglas en inglés, quienes de manera oficial presentó el término en 1992 como una contribución a la Cumbre Mundial sobre desarrollo Sostenible de Río de Janeiro a través de su publicación Changing Course, para el cumplimiento de la Agenda 21 en el sector privado; por lo que, allí se consideró la ecoeficiencia como una filosofía administrativa tal como lo manifiesta Jollands (2004), WBCSD (1997) y OECD (1998). En este orden de ideas, la ecoeficiencia es concebida como una propuesta fundamentalmente empresarial. Es una expresión acuñada por el Consejo Empresarial Mundial para el Desarrollo Sostenible (CEMDS), como una manera de embarcar de manera más proactiva a los sectores privados en una senda de sostenibilidad. Por eso, se le considera, al inicio, como equivalente a la producción limpia; que, sin embargo, se asocia más a políticas públicas y a temas de contaminación. Es por ello que, este concepto estuvo desde su origen más ligado a los temas del alivio o recuperación del deterioro ambiental (Leal, 2005).

No obstante, es importante tener en cuenta que la ecoeficiencia tiene otras características que se pueden sintetizar de la manera siguiente: enfatiza en la creación de valor, en la durabilidad, en el largo plazo, en objetivos para el mejoramiento continuo, vincula la excelencia ambiental con la excelencia empresarial y considera tanto el consumo como la producción sostenible (DeSimone \& Popoff, 1997). 
En torno al término producción más limpia, según el Programa de Naciones Unidas para el Medio Ambiente (PNUMA) se refiere a la aplicación continua de una estrategia integrada de prevención ambiental en los procesos, los productos y los servicios, con el objetivo de reducir riesgos para los seres humanos así como para el medio ambiente, incrementar la competitividad de la empresa para garantizar la viabilidad económica; mientras que el término ecoeficiencia es una estrategia corporativa, empresarial, cuyas raíces provienen del sector privado.

Con el pasar del tiempo, la frase ecoeficiencia ha sido sometida amuchas interpretaciones, pero Hinterberger y Stiller (1998) sostienen que, todas coinciden en un uso más eficiente de los recursos naturales. En cuanto al prefijo "eco", para unos, hace referencia a los asuntos ambientales (Jollands, 2004); mientras que para otros, "eco" se refiere tanto a lo ecológico como a lo económico (OECD, 1998). En síntesis, la ecoeficiencia mide la relación entre las salidas (outputs) y entradas (inputs) en un proceso productivo; por lo que, cuando más altas sean las salidas en un proceso para un determinado volumen de insumos, menores serán los insumos utilizados y más eficiente será el negocio (Burritt y Saka, 2006).

Para World Business Council for Sustainable Development (1997), la ecoeficiencia se define como proporcionar bienes y servicios a un precio competitivo, satisfaciendo las necesidades humanas y la calidad de vida, al tiempo que se reduce progresivamente el impacto ambiental y la intensidad de la utilización de recursos a lo largo del ciclo de vida, hasta un nivel compatible con la capacidad estimada que puede soportar el Planeta. Por su parte Mickwitz (2006) sostiene que "es la eficiencia con la cual los recursos ecológicos son usados para satisfacer las necesidades humanas." (pág. 67).

Dentro del mismo contexto, Núñez (2006), Leal (2005) y Bartolomeo (2003) comentan sobre los dos pilares imprescindibles de la ecoeficiencia. El primero, referido a reducir la sobreexplotación de los recursos naturales logrando un uso más sostenible de ellos. El segundo, disminuir la contaminación asociada a los procesos productivos. Sin duda 
estos dos pilares se complementan de manera equilibrada a razón de propender porque se utilice menos y se produzca limpiamente.

De la misma manera, el documento plantea los objetivos siguientes: a) Reducir el consumo de recursos, que incluye minimizar el consumo de agua, energía, materiales, tener en cuenta los usos del suelo, aumentar el reciclaje, la durabilidad del producto y cerrar el ciclo de los materiales. b) Reducir el impacto ambiental que, abarca minimizar las emisiones gaseosas, los vertidos líquidos, residuos sólidos, entre otros; también contempla el consumo racional de los recursos naturales. c) Suministrar más valor con el producto o servicio: significa más beneficios a los usuarios, por medio de la funcionabilidad, la flexibilidad y la modularidad del producto, entregando servicios adicionales y enfocándose en vender la solución a las necesidades de los clientes.

Al respecto, Bleischwitz (2003) presenta los tres estados de la ecoeficiencia en la cadena de producción: El primero se refiere al período de la toma de decisiones, es decir, es la etapa de las ideas y las expectativas sobre futuros desarrollos. El segundo señala el periodo de adaptación y el tercero es el de renovación, en el cual se ha superado la etapa de aprendizaje y aparecen nuevas innovaciones.

Sobre la base de las ideas planteadas, se puede advertir que el término ecoeficiencia es relativamente nuevo, que inicialmente se planteó como una filosofía administrativa para el sector privado pero que con el transcurrir del tiempo se le atribuyeron una serie de elementos que han cambiado la forma de gerenciar las organizaciones; es relevante resaltar que, la transformación del concepto viene en concordancia con la necesidad de articular acciones que garanticen la sostenibilidad de los recursos provenientes del planeta fundamentado en la sustentabilidad de la organización.

\section{Indicadores de la ecoeficiencia}

Según Courcelle (1998), los aspectos económicos, medioambientales, socioculturales y técnicos, se constituyen en los cuatro los factores clasificados, alrededor del cual se deben estructurar los indicadores que deben ser abordados por las organizaciones e 
instituciones, tanto a corto como a largo plazo, con el fin de apuntar sus procesos organizacionales en términos de ecoeficiencia (ver cuadro 1). En esta instancia, los aspectos más relevantes a corto plazo implican un cambio drástico en sus procedimientos y operaciones que seguramente van acompañados de una transformación en la gestión del conocimiento en cada uno de los miembros de la organización; por supuesto, es menester una inversión económica importante para ello.

\section{Cuadro 1.}

Factores requeridos en la estructuración de indicadores para la gestión ecoeficiente.

\begin{tabular}{|c|l|l|}
\hline Factor & \multicolumn{1}{|c|}{ Corto plazo } & \multicolumn{1}{c|}{ Largo Plazo } \\
\hline \multirow{5}{*}{ Económicos } & $\begin{array}{l}\text { Costes de inversión } \\
\text { Coste neto de operación } \\
\text { Coste neto total por tonelada } \\
\text { Coste neto anual } \\
\text { Mercado potencial }\end{array}$ & $\begin{array}{l}\text { Viabilidad a largo plazo de } \\
\text { búsqueda y ordenación de } \\
\text { operaciones futuras. }\end{array}$ \\
\hline \multirow{3}{*}{ Medioambientales } & $\begin{array}{l}\text { Calidad de reconversión de } \\
\text { material } \\
\text { El residuo } \\
\text { La polución } \\
\text { El ruido } \\
\text { El uso de recursos naturales }\end{array}$ & $\begin{array}{l}\text { Impacto global: pérdida de } \\
\text { biodiversidad, peligros } \\
\text { globales, deposiciones } \\
\text { ácidas, paisajismo. }\end{array}$ \\
\hline Sociales y culturales \\
& $\begin{array}{l}\text { Aceptación pública } \\
\text { Participación } \\
\text { Nivel de salud pública } \\
\text { Empleos }\end{array}$ & $\begin{array}{l}\text { Bienestar, la disponibilidad } \\
\text { de recursos } \\
\text { (materia y energía). }\end{array}$ \\
\hline Técnicos & Escala Flexibilidad & Futuro desarrollo potencial. \\
\hline
\end{tabular}

Fuente: Courcelle et al. (1998).

Desde otra perspectiva, las acciones ecoeficientes de una empresa deben contemplar indicadores generales y específicos, los cuales, en su mayoría son un esfuerzo voluntario por parte de las organizaciones interesadas en incluir la ecoeficiencia dentro de sus estrategias de desarrollo corporativo. De ahí que, los dos tipos de indicadores están basados en la fórmula que une las dimensiones económica y ambiental (Ver 
figura $N^{\circ} 1$ ). De manera que, la ecoeficiencia $(E)$, se obtiene a través de la razón entre los indicadores que hacen referencia al valor del servicio o producto $(\mathrm{V})$, así como su impacto ambiental (Al) acusada por la generación o uso de los productos y servicios a lo largo del ciclo de vida.

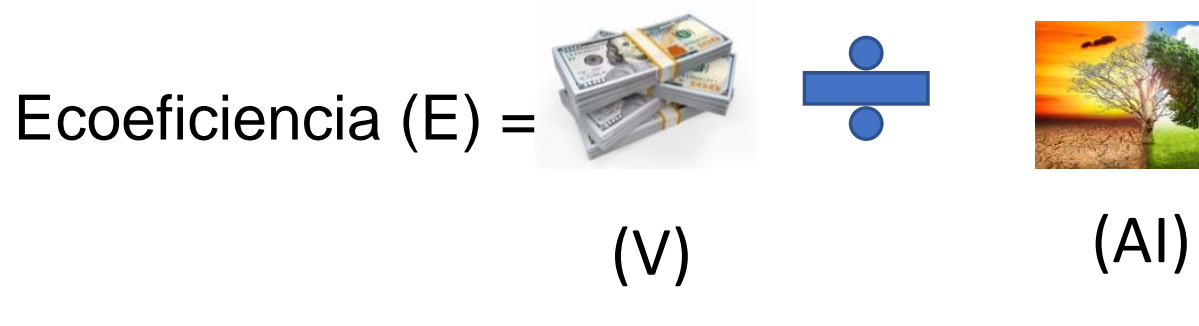

Figura 1.

Fuente: Erkko, Melanen y Mickwitz (2005).

Del mismo modo, Núñez (2006), sostiene que el impacto ambiental se ve entonces puesto en relación con los factores económicos, abarcando aspectos como materias primas, residuos, gasto de agua y volumen y tipo de aguas residuales, uso de energía eléctrica y combustibles fósiles, o emisiones a la atmósfera. En el numerador, el valor del producto o servicio puede estar representado por la cantidad de bienes o servicios producidos, las ventas netas, el monto de exportaciones e importaciones o el número de empleos directos generados.

Bajo esta perspectiva, la empresa que desee maximizar su ecoeficiencia podrá hacerlo aumentando el numerador (según la fórmula de ecoeficiencia) en mayor proporción que el denominador o disminuyendo este último. Pero, se podría estimar que resulta más conveniente plantear la minimización de la relación inversa, ya que bajo el contexto del Desarrollo Sostenible el objetivo debería orientarse a minimizar los impactos que a maximizar el valor de la producción (García, 2005); considerando que cada vez es más evidente que la búsqueda de la sostenibilidad y del desarrollo sostenible requiere integrar factores económicos, sociales, culturales, políticos y ecológicos (CNUMAD,1992; Gallopín et. al, 2001; Kates et. al, 2001). 
En otro orden de ideas, para abordar la gestión ambiental sostenible, resulta pertinente señalarlo como aquel término originado del concepto de desarrollo sostenible según el tesauro de la UNESCO. De ahí que, para Colby (1990) es el "campo que busca equilibrar la demanda de recursos naturales de la Tierra con la capacidad del ambiente natural, que responde a esas demandas con una base sustentable" (p. 5).

En este contexto, surgen elementos fundamentales en la búsqueda de la sustentabilidad ambiental, que tienen como principal objetivo; el conciliar las actividades humanas y el medio ambiente, a través de instrumentos que estimulen y viabilicen esa tarea, modificando el comportamiento del Hombre en su relación con la naturaleza, pues es productor de su degradación actual (Negrao, 2003)

Otra visión la aporta Guhl (2000), quien comenta que la gestión ambiental es "el manejo participativo de las situaciones ambientales de una región por los diversos actores, mediante el uso y la aplicación de instrumentos jurídicos, de planeación, tecnológicos, económicos, financieros y administrativos, para lograr el funcionamiento adecuado de los ecosistemas y el mejoramiento de la calidad de vida de la población dentro de un marco de sostenibilidad".

Desde la óptica empresarial, Manjarrés y Chirino (2020) sostienen que la Gestión Ambiental Sostenible en este ámbito, conlleva que ésta deberá estar orientada a cooperar con la conservación del medio ambiente, así como las condiciones de vida de las generaciones futuras. De ahí que, como concepto integrador, es superior al manejo ambiental, el cual debe dar respuesta a "cómo hay que hacer" para conseguir lo planteado, para lograr un desarrollo sostenible; en otras palabras, para alcanzar un equilibrio adecuado entre el desarrollo económico, crecimiento de la población, uso racional de los recursos, protección y la conservación del ambiente, entre otros.

En esta misma esfera, la ISO 14000 (2015) define el sistema de gestión ambiental como la parte del sistema general de gestión que incluye la estructura organizativa, la planificación de las actividades, las responsabilidades, las prácticas, los procedimientos, los procesos y los recursos para desarrollar, implantar, llevar a efecto, revisar y 
mantener al día la política ambiental. El sistema debe estar preparado para prevenir los impactos ambientales negativos, mediante la gestión de la: "identificación, evaluación, prevención, mitigación o compensación, constituyen el objeto de la gestión ambiental". Para el Ministerio del Medio Ambiente Colombiano, el desarrollo sostenible debe considerar cuatro premisas: 1) usar los recursos renovables de tal modo que el ritmo de extracción no sea mayor que el ritmo de regeneración natural; 2) mantener flujos de residuos al mismo nivel, o por debajo, de la capacidad de asimilación o de degradación de éstos al ambiente; 3) asegurar que a medida que mermen los recursos no renovables, tal reducción se vea compensada con el aumento de los recursos renovables y los sustitutos tecnológicos; 4) hacer posible el mantenimiento de una calidad de vida dada a partir de las existencias de recursos no renovables en disminución, permitiendo alcanzar una mayor eficiencia en el uso de los recursos naturales.

Partiendo de estas ideas planteadas, se puede decir que en las instituciones públicas, se presentan tres tipos de gestión ambiental: 1) Casual, integrada por políticas y planes que no tienen un objetivo ambiental, ejercen una incidencia en la protección del sistema ambiental, sobre todo del subsistema natural. 2) Sectorial, conformada por políticas y planes tendientes a la protección de algunos elementos ambientales o recursos naturales renovables. 3) Gestión ambiental propiamente dicha, integrada por políticas y planes que conciben el sistema ambiental como un todo, similarmente al enfoque sistémico.

De igual manera, Vega Mora,(2001) contribuye ampliando la clasificación aludida ya no solo de la gestión pública, sino también de la gestión empresarial, siendo un enfoque administrativo, que plantea, tres tipos de gestión ambiental; la Incidental, que ejecuta acciones aisladas, sin planeación estratégica, ni control; la Operacional, en donde se planifica, se ejecutan programas y proyectos ambientales, de manera incoherente y desarticulada, solo por cumplir una normativa; y la Sistémica, donde coexisten simultáneamente la planificación, la ejecución y el control; este tipo de gestión se 
caracteriza por ser previsiva en la planificación, eficiente en la ejecución, eficaz en el control, con un proceso de aprendizaje, retroalimentación y mejoramiento continuo. Es, en esencia, el precepto conceptual básico del enfoque sistémico.

Cabe resaltar, que Colby (1990), en cuanto a la gestión ambiental, identifica cinco elementos asociados a cinco paradigmas:

Economía de frontera. Es el paradigma dominante en los países industriales, en donde la naturaleza existe como un instrumento para beneficiar al Hombre, siendo explorada, manipulada y modificada por él, sin importar la manera, siempre para mejorar la calidad material de su vida.

Ecología profunda. El Hombre es colocado en una posición de subordinación a la naturaleza, resultando en: igualdad de las especies, reducción de la población humana, autonomía bio-regional, promoción de la diversidad ecológica y cultural, economía orientada al no crecimiento, fin de la dominación de la tecnología.

Protección Ambiental. Se institucionalizan los estudios ambientales para la valoración de impactos y beneficios en relación a la contaminación ambiental; emergiendo con ello agencias gubernamentales de protección ambiental.

Gestión de Recursos. Consideración negativa de contaminación que provoca la degradación del capital natural; el clima y los procesos de regulación son recursos fundamentales y vitales, que deben ser gerenciados por este enfoque. Parques y reservas son considerados recursos genéticos y elementos fundamentales como reguladores climáticos.

Ecodesarrollo. Es el paradigma más adecuado al futuro, la gestión ambiental es reorientada hacia la protección ambiental, con una economía abierta, termodinámicamente encajada dentro del ecosistema, parte del flujo de recursos biofísicos (energía, materiales y los ciclos de procesos ecológicos) sale del ecosistema para la economía, y la energía degradada (no utilizable) y otros subproductos (contaminación) fluyen de vuelta para el ecosistema. 
Los procesos de cambio de mentalidad ambiental, plasmados en políticas institucionales, dieron como respuesta unas herramientas que buscan mejorar el ambiente, a saber;

a) Evaluación de Impacto Ambiental. Originada con la promulgación del National Environmental Policy Act (NEPA), en los Estados Unidos, en 1969, e incorporada en otros países solamente después de la Conferencia de Estocolmo en 1972 (MAGRINI, 1990).

b) Programas de Monitoreo Ambiental. Comprende el seguimiento sistemático de la variación temporal y espacial de parámetros ambientales, posibilitando una evaluación constante y enfocada en los puntos neurálgicos que deben ser solucionados.

c) Auditoría ambiental. Según AMARAL (1993), es la concepción más difundida en la Comunidad Económica Europea. Es la herramienta de gestión que realiza una evaluación sistemática, documentada, periódica y objetiva del desempeño de una organización, de su sistema de gerencia y de los equipamientos destinados a la protección del medio ambiente

d) Análisis de riesgos. Consiste en la identificación de elementos y situaciones de una actividad cualquiera, mediante las siguientes fases; la identificación y clasificación de eventos peligrosos; la determinación de la frecuencia de ocurrencia utilizando cálculos de probabilidad; el análisis de los efectos y daños mediante modelos matemáticos; y la determinación de técnicas de control y mitigación

e) Due diligence. Es un instrumento asociado a las fusiones, adquisiciones de compañías o terrenos, referentes a los seguros ambientales, para descubrir potenciales obligaciones $\mathrm{y} / \mathrm{o}$ costos ambientales, o pasivo ambiental causados por el propietario anterior. 
Universidad Nacional Experimental Francisco de Miranda (UNEFM). Santa Ana de Coro. Venezuela

Diana Patricia Eljach-Hernandez; William Wannergg Castro-Castellanos

f) Programas de recuperación ambiental. Constituye un instrumento de planificación y gestión ambiental, que debe estar previsto desde las fases iniciales de un proyecto, con el objetivo de presentar soluciones para que el área impactada y degradada, presente nuevamente condiciones de equilibrio dinámico con su entorno, con vista de su futura utilización técnica y económicamente viables.

g) Programas de medidas de emergencia. Complementan los análisis de riesgo, involucran la formulación de acciones dirigidas a la atención de emergencias de tipo ambiental. La eficacia y la eficiencia estará determinada si contempla un programa de intervención, estudios de medidas preventivas, para minimizar daños y riesgos al medio ambiente, a los trabajadores y al área de influencia.

h) Programas de comunicación. Para Martínez Sánchez (1994), estos programas son los más aceptados por las empresas, pero a veces confundidos con otros programas. La imagen de la empresa se construye, "a través del diálogo y del respecto a los ciudadanos, incluyendo la comunidad en la que la empresa está instalada, la opinión pública de modo general y los agentes de los órganos gubernamentales" (Martínez Sánchez, 1994, p.70).

Retrocediendo en el tiempo, se recuerda el 2015 como el año del nacimiento de la Agenda 2030. Esta agenda, definido un modelo para alcanzar la prosperidad en un mundo sostenible en donde cada habitante desarrolle una vida productiva, apasionante y pacífica en nuestra casa, el planeta tierra. A escasos 10 años del 2030, es necesario abordar el cuestionamiento, ¿Las medidas que se están adoptando hasta hoy, han logrado cimentar las bases para alcanzar los Objetivos de Desarrollo Sostenible (ODS)?, al respecto, hay, hubo y de seguro habrá pronunciamiento.

Aunado a lo anterior, en el 2019, el Secretario General de la ONU hizo un llamado a todos los sectores de la sociedad a movilizarse para el logro de 3 estrategias 
fundamentales para conseguir los Objetivos de Desarrollo Sostenible. La primera estrategia, denominada acciones a nivel mundial, que han de promover un real liderazgo, recursos y soluciones innovadoras; la segunda estrategia, que gira en torno a realizar acciones a nivel local, que acerquen las políticas, presupuestos, y las instituciones de los gobiernos locales al logro de estos objetivos; y la ultima estrategia, que implica las acciones por parte de los individuos, en donde los jóvenes, la sociedad civil, los medios de comunicación, el sector privado, académicos, todos en conjunto generen un movimiento para el logro de las verdaderas las transformaciones que se necesitan para alcanzar los ODS.

También, cabe recordar, que la declaratoria de la ONU (2015) refiere: «Estamos resueltos a poner fin a la pobreza y el hambre en todo el mundo de aquí a 2030, a combatir las desigualdades dentro de los países y entre ellos, a construir sociedades pacíficas, justas e inclusivas, a proteger los derechos humanos y promover la igualdad entre los géneros y el empoderamiento de las mujeres y las niñas, y a garantizar una protección duradera del planeta y sus recursos naturales", señalaron los Estados en la resolución.»

Aquí, se hace necesario resaltar lo contemplado en los 17 objetivos del desarrollo sostenible (PNUD, 2016); esto para entender la importancia de su cumplimiento, desde lo general a lo específico en el tema tratado, la ecoeficiencia y su implementación en la gerencia de este milenio. De ahí que, para lograr el fin de la pobreza se debe evitar disminuir el $8 \%$ de caída en los indicadores, dado que esto provocaría regresar a la situación vivida en los años 30 , tal y como resalta la Universidad de las Naciones Unidas. Por lo menos 500 millones de personas entrarían a engrosar las filas del desempleo, aunado a la actual situación de pandemia, un lamentable retroceso de casi un siglo.

Con respecto a los objetivos de poner fin al hambre, logrando la seguridad alimentaria, mejorando la nutrición con la promoción de la agricultura sostenible y así alcanzar una vida sana, llena de bienestar para todos, sin distingo de edades. Solo será posible si se 
logra el crecimiento económico sostenido, inclusivo y sostenible, dando lugar a mejores empleos, productivos y decentes para todos, mediante el aumentando de la industrialización inclusiva, sostenible, la cual debe ir acompañada de la innovación e infraestructura, para que pueda incentivar las fuerzas económicas dinámicas y competitivas que generan el empleo y los ingresos.

Para ello, se hace necesario tener una educación inclusiva, equitativa, de calidad y que promueva las oportunidades de aprendizaje durante toda la vida para todos. Así se reducirían las desigualdades, permitiendo caminar todos juntos en igualdad de géneros, empoderando a las mujeres y las niñas, viviendo en ciudades con asentamientos humanos inclusivos, seguros, resilientes y sostenibles digno de sociedades justas, pacíficas e inclusivas.

También, es imperioso tener garantizada la disponibilidad de agua, su gestión sostenible y el saneamiento para todos, con acceso a energías asequible, seguras, sostenibles y enmarcadas dentro de la modernidad, con modalidades de consumo y producción sostenibles, gracias a las medidas tomadas para combatir el cambio climáticos y sus efectos; conservando los océanos, los mares, los recursos marinos gestionados de manera sostenible, así como los bosques, deteniendo e invirtiendo el proceso desertificación de las tierras y evitar la pérdida de biodiversidad. Todo lo anterior se podría convertir en una realidad si logramos revitalizar una inmensa Alianza Mundial para el Desarrollo Sostenible.

Sin embargo, hace falta más que simples retoricas en pro del medio ambiente; es necesario traer al tiempo presente eso de; "transformar el mundo en los próximos 15 años" para "librarlo de la pobreza extrema y del hambre que todavía hoy afligen a buena parte de la Humanidad". Palabras del gobierno español que quieren jalonar el cambio de mentalidad y sobre todo actuar en busca de esa meta, que no solo será de ellos, sino de todos. La tierra es el único hogar que se tiene, sino se cuida y se sigue haciendo lo mismo, el resultado será igual al que se ha visto hasta ahora. 
¿Y entonces cuál será el rol del sector productivo?, ante esta interrogante, surgen tres enfoques que se deben abordar: en primer término, la filantropía que permitirá mejorar las condiciones sociales y ambientales de los entornos circunvecinos de la organización, enmarcado en la responsabilidad social empresarial que aumenta su imagen en la sociedad. Seguidamente, la eficiencia operacional o atrayendo y reteniendo al talento. Finalmente, con un modelo de innovación en los productos y servicios en pro de los ODS y generador oportunidades de negocio. En este sentido, el protocolo del SDG* Compass (Ver figura 2), es una herramienta de apoyo para que las organizaciones puedan alinear sus estrategias, además de medir y gestionar su contribución, en pro de los ODS

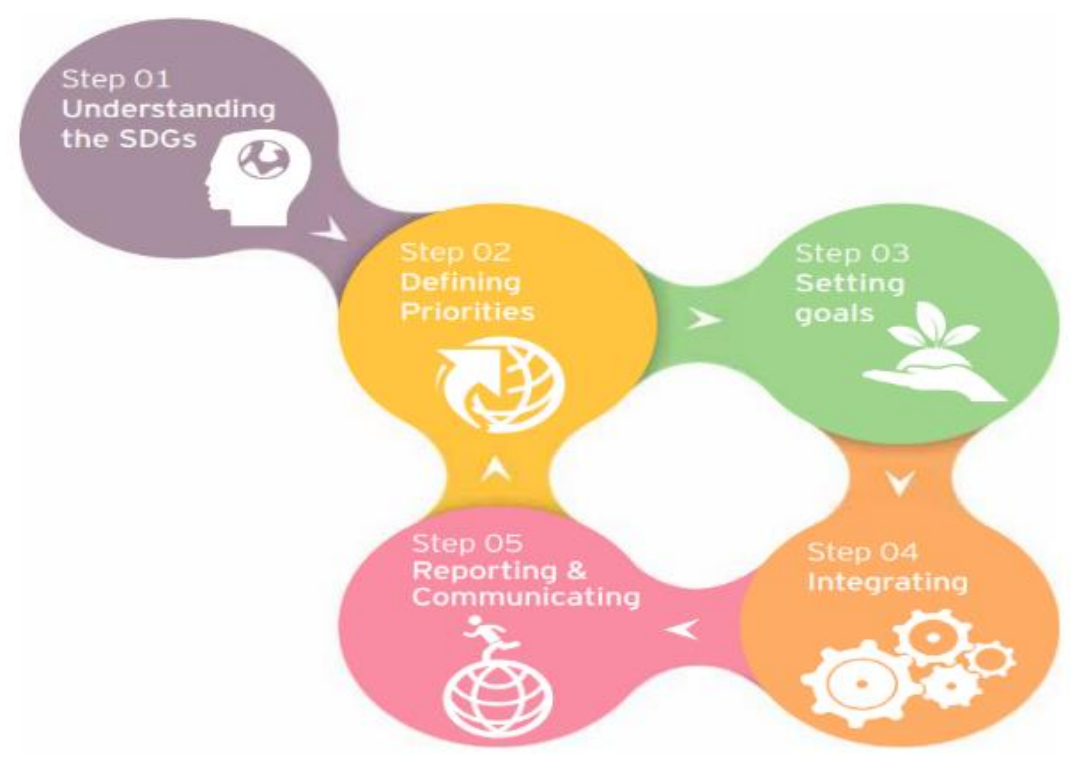

Figura 2. Figura SDG* Compass.

Fuente: SDG Compass (2015). 
Para acompañar a las organizaciones empresariales en busca de cumplir con los ODS, Global Reporting Initiative (GRI), The United Nations Global Compact (Pacto Global) y el World Business Council for Sustainable Development (WBCSD), desarrollaron el SDG Compass, un protocolo de gestión que tiene cinco etapas, las cuales buscan el aporte y fomento del compromiso público, para afinar estrategias y dar a conocer los avances. Este proceso es una secuencia lógica de etapas de la acción empresarial que buscan situar la sostenibilidad alineada a la estrategia corporativa (Ver figura 3).

A manera de inicio, la primera etapa implica que la organización y/o empresa penetre los ODS, entienda cuales son las oportunidades y responsabilidades que representan, descubra como impactan en la producción generando valores agregados; y, con ello, como puede aportar la compañía al logro de los ODS. En la segunda etapa se identifican impactos generados, origen y su alcance, los cuales se correlacionan con los ODS, seleccionando los indicadores para revisar su evolución, es decir la huella de la empresa. Utilizando metodologías de estándares mundiales, se verifica el dimensionamiento de los impactos, definiendo los puntos prioritarios de atención, análisis con un enfoque multivariable que incluya la magnitud, severidad y probabilidad de ocurrencia. En la tercera etapa, se establecen los objetivos y metas a alcanzar, limitando interna y externamente los impactos. Esto da origen a los programas de trabajo y acciones que hagan efectivo los objetivos en el tiempo.

La cuarta etapa es la implementación del plan de acción, en donde se asignan las tareas en toda la organización y a sus integrantes, asociados a los indicadores respectivos de seguimiento. Aquí es primordial el compromiso Sine Quanon de la Directiva para poder generar un compromiso de todos y cada uno de los miembros de la compañía. La quinta etapa, es la publicación de los avances en cuanto al desempeño y logros alcanzados. SDG Compass recomienda seguir estándares de reporte internacionales que incluyen información transversal de varias áreas de acción, laborales, comunitarios, ambientales. Por consiguiente, es importante comunicar: 1) Por qué es relevante y que impactos genera la compañía, 2) los objetivos y las actuaciones 
diseñadas, y 3) los sistemas de gestión implementados, para cada ODS inherente a la empresa.

\section{Guía de la acción empresarial para gestionar sistemas situando la sostenibilidad alineada a la estrategia corporativa}

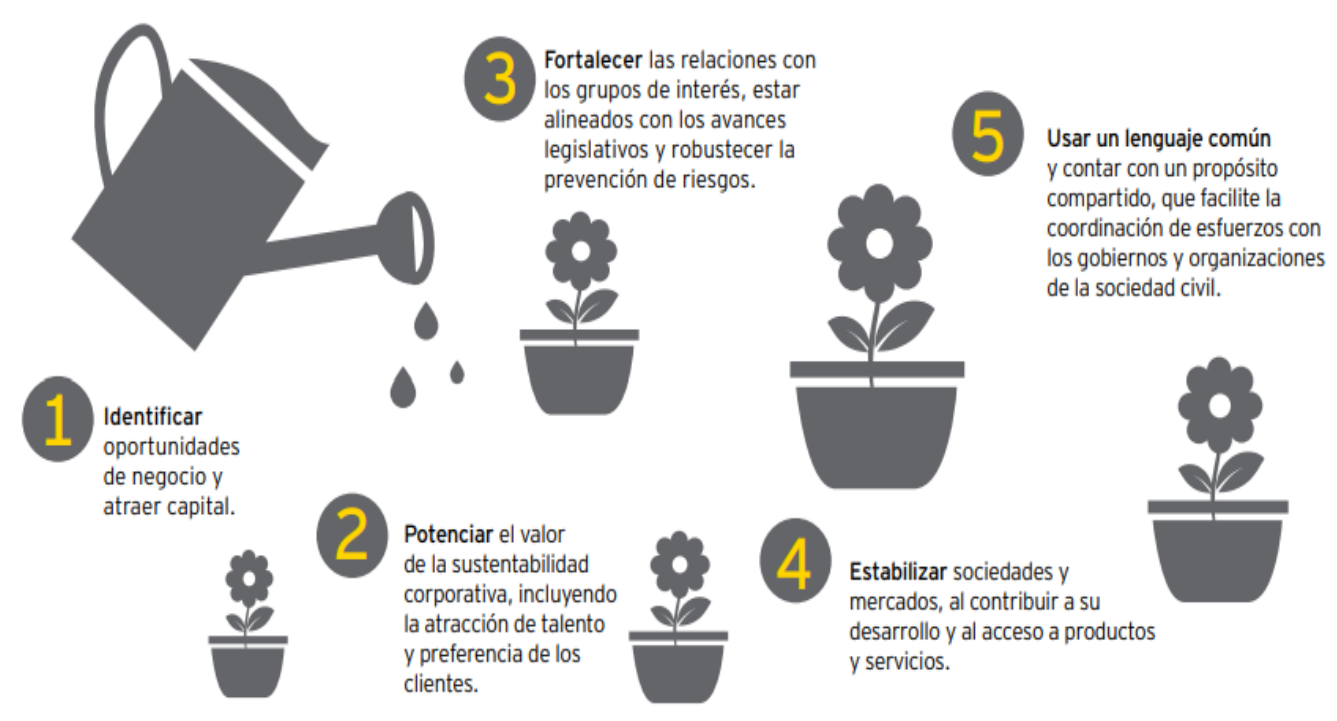

\section{Figura 3.}

Fuente: Adaptado de SDG Compass. The guide for business action on the SDGs (GRI, UN Global Compact \& WBCSD, 2015).

Ahora bien, ante los planteamiento hechos, desde diferentes trincheras académicas nacen diversas y disimiles preocupaciones por el desarrollo de la administración y su futuro inmediato, dando como resultado la situación de crisis producto del enquistamiento en la funcionalidad, mientras el mundo va al ritmo de los cambios y las innovaciones presentadas en las organizaciones emergente; muchas de las cuales, ya posicionadas, están amasando su capital, tal vez por la maximización del mismo, dejando de lado a su talento humano. Es hora de preparar, pensar o buscar alternativas que lleguen a armonizar todas las áreas y sentires de las empresas. 
Para Covey (2003), las cosas se administran, la gente se lidera, claro ejemplo de la tendencia de la administración, resultando la división de la administración de los recursos y la gestión de la gente. El gerente, debe ser capaz de entender en donde se está desarrollando como actor primordial, lo cual es el resultado del análisis y conocimiento de la procedencia y hacia dónde quiere llegar en un plazo determinado sin dejar las características de su entorno, las diferentes avances o estancamiento al respecto. Como gerentes, las personas realizan las funciones gerenciales de planear, organizar, integrar personal, dirigir y controlar. En la alta gerencia las habilidades conceptuales, de diseño y humanas son especialmente valiosas, mientras que las técnicas son relativamente poco necesarias.

Con respecto al enfoque de las funciones gerenciales, Mintzberg (2016) de la Universidad McGill, producto de sus observaciones sobre el quehacer de los gerentes enuncia que los ejecutivos no realizan las funciones gerenciales clásicas de planear, organizar, comandar, coordinar y controlar; por lo contrario, se dedican a una variedad de actividades definidas en una serie de diez funciones: funciones interpersonales (de figura central); de líder, de enlace (en particular con personas de fuera); funciones informativas, de receptor, de difusor, de vocero; funciones de decisión, de emprendedor, de encargado de disturbios, de asignar recursos, de negociador con varias personas y grupos de personas (Koonzt, 2012).

\section{REFLEXIONES FINALES}

A manera de reflexión, se puede enunciar que los investigadores resaltan la importancia de implementar procesos de ecoeficiencia desde la gerencia con el propósito no solo de salvaguardar los recursos del planeta generando acciones amigables con el ambiente, si no que resulta vital para las organizaciones la reducción de costos de operaciones, la efectividad en la cadena de suministro, el fortalecimiento de la estrategia corporativa, la implementación de estándares nacionales e internacionales, la apertura de nuevos mercados entre otros. 
Cuando recordamos la frase del Friedman(1970): "El directivo de una corporación es un empleado de los dueños del negocio, tiene la responsabilidad con sus empleadores, en donde debe manejar el negocio de acuerdo a sus deseos; el cual, normalmente es ganar el máximo dinero posible respetando las reglas básicas de la sociedad". Es aquí donde se necesitan líderes con moral, conscientes del papel primordial que juegan sus decisiones para el entorno ambiental, pues, situaciones como el cambio climático puede traer graves consecuencias como; la escasez de recursos naturales, la disponibilidad de alimentos y la pobreza.

Las empresas que han entendido que su accionar tiene límites buscan mantener el equilibrio climatológico y la utilización sustentable de recursos ambientales; además, su original preocupación por el entorno social, hacen que prosperen a largo plazo, aumentando su imagen, su credibilidad e institucionalidad en la sociedad. Para esto es imprescindible que posean algunas características tales como; entendimiento de los riesgos del cambio climático, trabajar la incertidumbre, construir asociaciones y relaciones para reforzar su estrategia, fomentar el liderazgo colaborativo, entre otras. Solo así, pueden alcanzar la integración con su entorno social, mediante relaciones basadas en lo ético-axiológico, y con su entorno físico mediante una correlación equilibrada con la naturaleza.

Hoy, más que nunca, se hace importante la creación de un entorno de virtuosismo empresarial, en dónde las aristas de la productividad y la competitividad estén de la mano de los avances de la era tecnológica, en defensa y protección de los recursos naturales, además de impulsar un nuevo modelo de gerencia capaz de generar escenarios innovadores y sujetos organizacionales con pensamiento creativo y transformador, preservando la biodiversidad en marco de un crecimiento económico que logre satisfacer las necesidad propias del mercado sin menoscabo de la calidad de vida de la sociedad. Entonces, se podría concluir diciendo que esta es una invitación a reflexionar sobre como los actos humanos afectan las generaciones futuras; por tanto, como individuos y como organización hay que abordar la integración de las áreas 
económicas, tecnológicas, ambiental y social en total armonía para la pervivencia humana y del planeta.

\section{REFERENCIAS CONSULTADAS}

Aldana Zavala, J. J. (2019). La competencia epistemológica en el investigador social universitario venezolano. [Epistemological competence in the Venezuelan. university social researcher]. Praxis, 15(1), 103-115. https://doi.org/10.21676/23897856.3091.

AMARAL, S. P. (1993). Auditoría Ambiental: Uma Ferramenta de Gestão Ambiental nas Empresas. Saneamento Ambiental. n.25, p.40-50. Disponible en: https://estrucplan.com.ar/gestion-ambiental-segunda-parte/

Ávila., J. (2012). Redes sociales y análisis de redes: Aplicaciones en el contexto comunitario y virtual. Corporación Universitaria Reformada. Barranquilla. Azul y Violeta Editores Ltda. Disponible en: https://dialnet.unirioja.es/descarga/libro/511130.pdf.

Bartolomeo, M. (2003). Eco-efficient producer services. Journal of Cleaner Production.

Bleischwitz, R. (2003). Cognitive and institutional perspectives of ecoefficiency.Ecological Economic.Vol.46.

Burritt, R. L. y Saka, C. (2006). Environmental management accounting applications and Ecoefficiency: Case studies from Japan. Journal of Cleaner Production. Vol. 14, p. 1262-1275. Disponible en https://n9.cl/qe7a

CNUMAD (1992). Conferencia de las Naciones Unidas sobre el Medio Ambiente y el Desarrollo (CNUMAD). Naciones Unidas. Disponible en https://n9.cl/w2hh5

Courcelle, C.; Kestemont, M; Tyteca, D. (1998). Assessing the economic andenvironmental performance of municipal solid waste collection and sortingprogrammes. Waste Management \& Research, v.16, n.3, p.253-263. https://cutt.ly/RgAFF3H

Colby. E. (1990). Environmental Management in Development: The Evolution of Paradigns. World Bank Discussions Papers. n.80

Comisión Mundial del Medio Ambiente y del Desarrollo. (1988). Nuestro Futuro Común. Madrid: Alianza. https://n9.cl/bj6d5 
Corvey, S. (2003). Los 7 Hábitos de la Gente Altamente Efectiva: la revolución ética en la vicia cotidiana y en la empresa. Paidos. Buenos Aires. Disponible en: https://n9.cl/iy8tw

DeSimone, L. and F. Popoff. (1997). Eco-efficiency; the business link to sustainable development. Cambridge.

Erkko, S.; Melanen, M.; Mickwitz, P. (2005). Eco-efficiency in the finnish EMAS reports-a buzz word? Journal of Cleaner Production, p.799-813. https://cutt.ly/RgAFF3H.

Friedman, M., (1970).The Social Responsibility of Business is to_Increase its Profi ts. The New York Times Magazine. Disponible en: https://n9.cl/7b60

Gallopín, G.C. (1997), Branching Futures and Energy Projections. Renewable Energy for Development I0. Environmental and Sustainability Indicators and the Concept of Situational Indicators. A Systems Approach, Environmental Modelling \& Assessment I. I0I-II7. Disponible en: https://n9.cl/w2hh5

García, A. (2005). Breve historia de la educación ambiental: del conservacionismo hacia el desarrollo sostenible. En Revista Futuros. № 12 . Vol 3. http://www.revistafuturos.info/futuros 12/hist ea.htm

Gulh, E. (2000). Vida y Región. Citado por Ministerio del Medio Ambiente (Colombia). Sigam. Tomo 1.

Hinterberger, F. y Stiller, H. (1998). Energy and Material Flow. En S. Ulgiati (Ed). Advances in Energy Studies: Energy Flow in Ecology and Economy.p.275-286. Italy: Musis Publisher. https://core.ac.uk/download/pdf/132826984.pdf

Jollands, N. (2004). Aggregate ecoefficiency indices for New Zealand: a principal components analysis. Journal of Environmental Management. No. 73. https://www.eumed.net/rev/delos/20/ecoeficiencia.html

Kates, R.W., W.C. Clark, R. Corell, J. M. Hall, C.C. Jaeger, I. Lowe, J.J. McCarthy, H.J. Schellnhuber, B. Bolin, N.M. Dickson, S. Faucheux, G.C. Gallopin, A. Gruebler, B. Huntley, J. Jager, N.S. Jodha, R.E. Kasperson, A. Mabogunje, P. Matson, H. Mooney, B. Moore III, T. O'Riordan, y U. Svedin. (200I) Sustainability science. Science. 64I-642. 
Kliksberg, Bernardo (2000). El rol del capital social y de la cultura en el proceso de desarrollo. Capital social y cultura: claves estratégicas para el desarrollo. Banco Interamericano de Desarrollo, Washington DC. Disponible en https://n9.cl/46fd8

Kramer, M. R., \& Porter, M. E. (2002). La ventaja competitiva de la filantropía corporativa. Harvard Business Review, 80(12), 49-62. Disponible en https://n9.cl/rfkc.

Koonzt, H. (2012). Administración: una perspectiva global y empresarial. McGraw-Hill. Disponible en: https://cutt.ly/mgSRBhf.

Leal, J. (2005). Ecoeficiencia: Marco de analisis, Indicadores y Experiencias. División de Desarrollo Sostenible y Asentamientos Humanos de la Comisión Económica para América Latina y el Caribe (CEPAL), Naciones Unidas, Santiago de Chile.

Magrini, A. 1990. A avaliaçâo de impactos ambientais. Aspectos Técnicos e Económicos. Ipea, Brasilia. p. 238. Disponible en: https://cutt.ly/LgSQ1Gq

Manjarres-Mejia, A., \& Chirino-García, R. (2020). Logística verde: Reto Gerencial para el manejo de la Gestión Ambiental Sostenible. CIENCIAMATRIA, 6(11), 4-21. https://doi.org/10.35381/cm.v6i11.309.

Martínez Sánchez, F. (1994). Investigación y Nuevas Tecnologías de la Comunicación en la Enseñanza: El Futuro Inmediato. Pixel - Bit, 2, 3-17. Disponible en: https://n9.cl/r7yu

Mintzberg, H. (2016). Las 10 funciones gerenciales de Mintzberg. Universidad McGill. Disponible en: https://cutt.ly/wgSRfTM

Milckwitz, P.; Melanen, M.; Rosenströn, U.; Seppälä, J. Regional (2006). Eco efficiency indicators: a participatory approach. Journal of Cleaner Production, v. 14, p. 16031611. https://n9.cl/i454b

Negräo, C. Rachel. (2003). Gestión Ambiental: II Curso Internacional de Aspectos Geológicos de Protección Ambiental. Instituto de Geociencias de la UNICAMP. Brasil.

Normas ISO 1400. (2015). Principios y fundamentos de la Gestión Ambiental. Disponible en: https://n9.cl/t1e1f 
Nuñez, G. (2006). El Sector Empresarial en la Sostenibilidad Ambiental: Ejes de interacción. División de Desarrollo sostenible y Asentamientos Humanos de la Comisión Económica para América Latina y el Caribe (CEPAL) Naciones Unidas, Santiago de Chile.

OECD. (1998). Organization for Economic Co-operation and Development. Ecoefficiency. Disponible en https://dialnet.unirioja.es/servlet/articulo?codigo=5472521.

Organización de las Naciones Unidas. (1992). Informe de la conferencia de las naciones unidas sobre el medio ambiente y el desarrollo. Rio de Janeiro. Brasil.

Organización de las Naciones Unidas. (2015). Transformar Nuestro Mundo: La Agenda 2030 para el Desarrollo Sostenible. Nueva York. Disponible en: https://cutt.ly/igSEoqy.

Palella, S y Martins, P. (2012). Metodología de la investigación cualitativa. 3era edición. FEDUPEL. Caracas-Venezuela.

Programa de las Naciones Unidas para el Desarrollo PNUD. (2016). Objetivos de Desarrollo Sostenible. Recuperado de http://bit.ly/2ZNDQV8.

Schaltegger, S. y Sturm, A. (1990). Ecological Rationality: Starting Point for the Design of Ecological oriented Management Instruments. The Company, 4, 273-290. https://core.ac.uk/download/pdf/132826984.pdf

SDG Compass. (2015). The guide for business action on the SDGs. GRI, UN Global Compact \& WBCSD. Disponible en: https://n9.cl/pzapx

Schmidheiny S. (1992). Changing Course. MIT Press. Cambridge: MA. Disponible en: https://www.redalyc.org/pdf/5217/521751975003.pdf

Vega Mora., L. (2001). Gestión ambiental sistémica. Bogotá, Panamericana, Formas e Impresos S.A. Disponible en: https://cutt.ly/zgSTPKL.

WBCSD. (1997). Eco-efficiency and Cleaner Production. World Business council for Sustanaible Development. 
CIENCIAMATRIA

Revista Interdisciplinaria de Humanidades, Educación, Ciencia y Tecnología

Año VI. Vol. VI. N 1. Edición Especial. 2020

Hecho el depósito de ley: pp201602FA4721

ISSN-L: 2542-3029; ISSN: 2610-802X

Universidad Nacional Experimental Francisco de Miranda (UNEFM). Santa Ana de Coro. Venezuela

Diana Patricia Eljach-Hernandez; William Wannergg Castro-Castellanos

(C2020 por el autor. Este artículo es de acceso abierto y distribuido según los términos y condiciones de la licencia Creative Commons Atribución-NoComercial-Compartirlgual 4.0 Internacional (CC BY-NC-SA 4.0)

(https://creativecommons.org/licenses/by-nc-sa/4.0/). 